\title{
Development and Validation of Pre- and Post-Operative Models to Predict Recurrence After Resection of Solitary Hepatocellular Carcinoma: A Multi-Institutional Study
}

This article was published in the following Dove Press journal:

Cancer Management and Research

\author{
Ming-Yu $\mathrm{Wu}^{\mathrm{l}, *}$ \\ Qian Qiaol,* \\ Ke Wang ${ }^{2-4}$ \\ Gu-Wei ji ${ }^{2-4, *}$ \\ Bing Cai ${ }^{1}$ \\ Xiang-Cheng $\mathrm{Li}^{2-4}$ \\ 'Department of Hepatobiliary Surgery, \\ Wuxi People's Hospital, Wuxi, People's \\ Republic of China; ${ }^{2}$ Hepatobiliary Center, \\ The First Affiliated Hospital of Nanjing \\ Medical University, Nanjing, People's \\ Republic of China; ${ }^{3}$ Key Laboratory of \\ Liver Transplantation, Chinese Academy \\ of Medical Sciences, Nanjing, People's \\ Republic of China; ${ }^{4} \mathrm{NHC}$ Key Laboratory \\ of Living Donor Liver Transplantation \\ (Nanjing Medical University), Nanjing, \\ People's Republic of China \\ *These authors contributed equally to \\ this work
}

Background: The ideal candidates for resection are patients with solitary hepatocellular carcinoma (HCC); however, postoperative recurrence rate remains high. We aimed to establish prognostic models to predict HCC recurrence based on readily accessible clinical parameters and multi-institutional databases.

Patients and Methods: A total of 485 patients undergoing curative resection for solitary HCC were recruited from two independent institutions and the Cancer Imaging Archive database. We randomly divided the patients into training $(n=323)$ and validation cohorts $(n=162)$. Two models were developed: one using pre-operative and one using pre- and postoperative parameters. Performance of the models was compared with staging systems.

Results: Using multivariable analysis, albumin-bilirubin grade, serum alpha-fetoprotein and tumor size were selected into the pre-operative model; albumin-bilirubin grade, serum alphafetoprotein, tumor size, microvascular invasion and cirrhosis were selected into the postoperative model. The two models exhibited better discriminative ability (concordance index: 0.673-0.728) and lower prediction error (integrated Brier score: 0.169-0.188) than currently used staging systems for predicting recurrence in both cohorts. Both models stratified patients into low- and high-risk subgroups of recurrence with distinct recurrence patterns.

Conclusion: The two models with corresponding user-friendly calculators are useful tools to predict recurrence before and after resection that may facilitate individualized management of solitary HCC.

Keywords: hepatocellular carcinoma, resection, recurrence, survival, modelling

\section{Introduction}

Hepatobiliary Center, The First Affiliated Hospital of Nanjing Medical University, 300 Guangzhou Road, Nanjing 210029 , People's Republic of China

Tel +8618951999088

Fax +862568136450

Email drxcli@njmu.edu.cn

Bing Cai

Department of Hepatobiliary Surgery,

Wuxi People's Hospital, 299 Qingyang

Road, Wuxi 214023, People's Republic of China

Tel +86 |3358| | $207 \mid$

$\mathrm{Fax}+8651082700778$

Email wxphcb@163.com
Hepatocellular carcinoma (HCC) is ranked as the sixth most common cancer worldwide and often occurs in patients with chronic viral hepatitis and cirrhosis. ${ }^{1}$ Theoretically, liver transplantation offers the possibility to simultaneously cure the tumor and the underlying cirrhosis, whereas demand for organs far exceeds supply. ${ }^{1,2}$ Liver resection represents a rapidly executable surgical procedure and solitary HCC at an early stage, regardless of tumor size, in patients with well-preserved liver function represents the mainstay indication for resection; unfortunately, $\mathrm{HCC}$ recurrence due to dissemination or de novo tumor complicates $70 \%$ of cases at 5 years. $^{1-3}$

Although there is no accepted neoadjuvant or adjuvant option to reduce the risk of HCC recurrence, patients at high risk for recurrent disease are potential 
candidates for future clinical trials. ${ }^{1-3}$ On the other hand, follow-up protocol for patients with surgically resected HCC should optimize early detection of recurrence in high-risk patients, minimize harms from follow-up tests in low-risk patients, and remain cost-effective. ${ }^{4}$ Currently, several HCC staging algorithms, such as Barcelona Clinic Liver Cancer (BCLC), Hong Kong Liver Cancer (HKLC), Cancer of the Liver Italian Program (CLIP) and American Joint Committee on Cancer (AJCC) tumor-nodemetastasis (TNM) systems, have been proposed for prognostic prediction paired with treatment allocation. ${ }^{2}$ Nevertheless, their prognostic performances on classifying postoperative recurrence are barely satisfactory and patients with HCC do not recur through the evolutionary stages of this disease after resection. ${ }^{1}$ Recently, a few statistical models have been developed to predict HCC recurrence; however, none of them have been specifically established for patients with solitary lesion at an early stage, who are the ideal candidates for resection. ${ }^{5,6}$ Shen et $\mathrm{al}^{7}$ have proposed a prognostic model to provide risk estimation of postoperative recurrence for solitary HCC, but a single-institution database per se is no guarantee for generalizability. It is reasonable and necessary to seek well-established and validated clinical prognostic models tailored to predict individual recurrence risk for solitary HCC patients.

This study aimed to develop and validate prognostic models to predict tumor recurrence for surgically treated patients with solitary HCC at an early stage based on readily accessible clinicopathological parameters and multi-institutional databases.

\section{Patients and Methods}

\section{Patients and Study Design}

All patients who underwent curative resection for solitary HCC between June 2009 and December 2016 were recruited from 2 independent institutions (Institution 1: The First Affiliated Hospital of Nanjing Medical University; Institution 2: Wuxi People's Hospital). Eligible patients with solitary $\mathrm{HCC}$ from the Cancer Imaging Archive (TCIA; https://www.cancerimagingarchive.net/) were also identified. Inclusion criteria were patients with histopathologically confirmed solitary HCC who were treated with curative resection and follow-up data available. Exclusion criteria were patients with macroscopic vascular invasion or extrahepatic metastasis, any treatment (i.e., previous liver resection, local ablation, transarterial chemoembolization) before surgery, resection for ruptured tumor, and clinicopathological data incomplete. A total of 485 accrued patients were enrolled in this study, including 377 patients from Institution 1, 78 patients from Institution 2 and 30 patients from TCIA. We randomly divided them into a training cohort $(\mathrm{n}=323)$ and an independent validation cohort $(n=162)$ in a 2:1 ratio. This study was conducted in accordance with the Declaration of Helsinki. The institutional review boards of the participating institutions approved this study protocol and written informed consent was obtained from all patients.

Clinicopathological parameters were reviewed and collected according to patients' records. The albumin-bilirubin (ALBI) score was calculated as the following equation: score $=0.66 \times \log _{10}$ bilirubin $-0.085 \times$ albumin, where bilirubin is in $\mu \mathrm{mol} / \mathrm{L}$ and albumin in $\mathrm{g} / \mathrm{L} .{ }^{8}$ ALBI grade was defined as follows: score $\leq-2.60$ (ALBI grade 1), score more than -2.60 to $\leq-1.39$ (ALBI grade 2), and score $>$ 1.39 (ALBI grade 3). ${ }^{8}$ Tumor size was recorded as the largest diameter on transverse contrast-enhanced images before surgery. Pathological features, such as degree of tumor differentiation, microvascular invasion (MVI), and cirrhosis, were documented. MVI was defined as tumor within a vascular space lined by endothelium that was identifiable only on microscopy. ${ }^{9}$

\section{Follow-Up and Survival Analysis}

Patients underwent surveillance with alpha-fetoprotein (AFP), liver function, chest and abdominal imaging every 3 months for the first 2 years and every 6 months thereafter at Institution 1 and 2 according to the recommendation of the European Society for Medical Oncology. ${ }^{10}$ Follow-up data of patients in TCIA were obtained via the Cancer Genome Atlas public database. Recurrence-free survival (RFS) was defined as time from surgery to first tumor recurrence/metastasis, which was confirmed by computed tomography or magnetic resonance imaging. Patients with no documented recurrence were censored at the date last seen free of recurrence.

\section{Model Development and Validation}

Multivariable Cox proportional hazards regression model was built on variables with attainment of statistical significance in univariable analysis and by using stepwise backward selection process with Akaike information criteria (AIC) as the stopping rule. We constructed two models to predict postoperative recurrence of HCC using the training cohort: the pre-operative model (model-pre) was 
based on parameters available before surgery; the postoperative model (model-post) included pathological results in addition to the aforementioned parameters. The proportional hazards assumption of the models was tested by scaled Schoenfeld residuals. The two models were then validated in an independent dataset.

\section{Statistical Analysis}

Statistical analysis was performed using $\mathrm{R}$ software version 3.4.4 with $\mathrm{R}$ packages listed in Supplementary Method. Pearson's chi-square test or Fisher's exact test were used for categorical variables, and two-sample Student's $t$-test or Mann-Whitney $U$-test were used for numerical variables, when appropriate. To reduce the effect of small discrepancies, AFP value was normalized with a natural logarithm transformation. Model discrimination was measured by concordance index (C-index) and time-dependent area under the receiver operating characteristic curve (tdAUC). The larger C-index and tdAUC, the more accurate was the prognostic prediction. Model fit was assessed by calibration plots via 1000 bootstrap resamples. Prediction error was summarized with the integrated Brier score (IBS), which represents the differences between actual events and predicted probabilities. ${ }^{11}$ The IBS was evaluated using "Boot632plus" split method with 1000 iterations while the lower IBS represents a more accurate model. Decision curve analysis (DCA) was used to determine the net benefits of model-assisted decisions at different threshold probabilities. We selected the optimum cutoff score for continuous prognostic factors using the X-tile software (version 3.6.1; Yale University). ${ }^{12}$ Recurrence and survival probabilities were estimated by the Kaplan-Meier method and compared using the Log rank test. Statistical significance was set at 0.05 .

\section{Results}

\section{Patient Characteristics}

The clinicopathological characteristics of patients in the training and validation cohorts are shown in Table 1. There were no significant differences between two cohorts with respect to any clinicopathological covariate. The median follow-up time was 48.4 (range, 1.9-109.2) months and 59.9 (range, 4.2-110.3) months in the training and validation cohorts, respectively. The median RFS of the training cohort was 56.1 months, with postoperative 2-and 5-year recurrence rates of $31.1 \%$ and $53.2 \%$, respectively; the median RFS of the validation cohort was 56.6 months,
Table I Patient Characteristics

\begin{tabular}{|c|c|c|c|}
\hline Variable & $\begin{array}{l}\text { Training Cohort } \\
(n=323)\end{array}$ & $\begin{array}{l}\text { Validation } \\
\text { Cohort }(n=162)\end{array}$ & $P$ value \\
\hline Age (years) & $58(49-66)$ & $58(49-65)$ & 0.904 \\
\hline $\begin{array}{l}\text { Sex } \\
\qquad \text { Male } \\
\text { Female }\end{array}$ & $\begin{array}{l}24 I(74.6) \\
82(25.4)\end{array}$ & $\begin{array}{l}116(71.6) \\
46(28.4)\end{array}$ & 0.478 \\
\hline $\begin{array}{l}\text { Etiology } \\
\text { Hepatitis B virus } \\
\text { Hepatitis C virus } \\
\text { Other }\end{array}$ & $\begin{array}{l}23 \mid(7 \mid .5) \\
16(5.0) \\
76(23.5)\end{array}$ & $\begin{array}{l}122(75.3) \\
4(2.5) \\
36(22.2)\end{array}$ & 0.385 \\
\hline $\begin{array}{l}\text { Bilirubin }(\mu \mathrm{mol} / \mathrm{L}) \\
\text { Albumin }(\mathrm{g} / \mathrm{dL}) \\
\text { Creatinine }(\mu \mathrm{mol} / \mathrm{L}) \\
\text { Platelet count }\left(\times 10^{9} / \mathrm{L}\right)\end{array}$ & $\begin{array}{l}15.8(12.0-21.1) \\
40.7(37.1-43.2) \\
71.8(61.0-83.5) \\
141.0(95.0-193.0)\end{array}$ & $\begin{array}{l}15.5(12.0-20.5) \\
40.0(36.1-43.0) \\
71.7(60.5-84.4) \\
147.0(95.8-207.3)\end{array}$ & $\begin{array}{l}0.704 \\
0.173 \\
0.972 \\
0.411\end{array}$ \\
\hline $\begin{array}{l}\text { Child-Pugh class } \\
\text { A } \\
\text { B }\end{array}$ & $\begin{array}{l}307(95.0) \\
16(5.0)\end{array}$ & $\begin{array}{l}153(94.4) \\
9(5.6)\end{array}$ & 0.777 \\
\hline $\begin{array}{l}\text { ALBI grade } \\
\qquad \begin{array}{l}1 \\
2 \text { or } 3\end{array}\end{array}$ & $\begin{array}{l}193(59.8) \\
130(40.2)\end{array}$ & $\begin{array}{l}92(56.8) \\
70(43.2)\end{array}$ & 0.532 \\
\hline $\begin{array}{l}\text { Serum AFP }(\mathrm{ng} / \mathrm{mL}) \\
\text { Tumor size } \\
\text { (imaging, } \mathrm{cm} \text { ) }\end{array}$ & $\begin{array}{l}26.1(3.8-468.1) \\
4.7(3.1-6.8)\end{array}$ & $\begin{array}{l}33.5(4.7-47 \mid .2) \\
4.1(2.7-6.2)\end{array}$ & $\begin{array}{l}0.661 \\
0.109\end{array}$ \\
\hline $\begin{array}{l}\text { Edmondson-Steiner } \\
\text { grade } \\
\text { I-II } \\
\text { III-IV }\end{array}$ & $\begin{array}{l}215(66.6) \\
108(33.4)\end{array}$ & $\begin{array}{l}113(69.8) \\
49(30.2)\end{array}$ & 0.479 \\
\hline $\begin{array}{l}\text { Microvascular } \\
\text { invasion } \\
\text { Absent } \\
\text { Present }\end{array}$ & $\begin{array}{l}246(76.2) \\
77(23.8)\end{array}$ & $\begin{array}{l}|3|(80.9) \\
31(19.1)\end{array}$ & 0.240 \\
\hline $\begin{array}{l}\text { Liver cirrhosis } \\
\text { Absent } \\
\text { Present }\end{array}$ & $\begin{array}{l}154(47.7) \\
169(52.3)\end{array}$ & $\begin{array}{l}81(50.0) \\
8 \mid(50.0)\end{array}$ & 0.629 \\
\hline
\end{tabular}

Notes: Continuous variables are presented as median (interquartile range); categorical variables are presented as $n(\%)$.

Abbreviations: ALBI, albumin-bilirubin; AFP, alpha-fetoprotein.

with postoperative 2 -and 5-year recurrence rates of $27.2 \%$ and $52.9 \%$, respectively. RFS was similar between the two study cohorts $(P=0.747$, Log rank test $)$.

\section{Development and Validation of Prognostic Models}

As shown in Figure S1, among 14 clinicopathological parameters analyzed in the training cohort, 6 were found to be significantly associated with postoperative recurrence in the univariable Cox regression analysis. Stepwise multivariable 
Table 2 Multivariable Cox Regression Results for RFS in the Training Cohort

\begin{tabular}{|c|c|c|c|c|c|c|}
\hline \multirow[t]{2}{*}{ Intercept and Variable } & \multicolumn{3}{|c|}{ Model-pre } & \multicolumn{3}{|c|}{ Model-post } \\
\hline & $\boldsymbol{\beta}$ & Hazard Ratio $(95 \% \mathrm{Cl})$ & P value & $\boldsymbol{\beta}$ & Hazard Ratio $(95 \% \mathrm{Cl})$ & $P$ value \\
\hline ALBI grade & 0.387 & $1.473(1.075-2.017)$ & 0.016 & 0.293 & $1.340(0.974-1.845)$ & 0.072 \\
\hline In (Serum AFP) & 0.177 & $1.193(1.117-1.274)$ & $<0.001$ & 0.126 & $1.134(1.060-1.214)$ & $<0.001$ \\
\hline Tumor size & 0.125 & $1.133(1.083-1.186)$ & $<0.001$ & 0.080 & $1.083(1.029-1.141)$ & 0.003 \\
\hline Microvascular invasion & NA & NA & NA & 1.008 & $2.741(1.869-4.019)$ & $<0.001$ \\
\hline Liver cirrhosis & NA & NA & NA & 0.300 & $1.350(0.97|-| .876)$ & 0.074 \\
\hline Akaike information criterion & \multicolumn{3}{|c|}{1646.38} & \multicolumn{3}{|c|}{1617.78} \\
\hline
\end{tabular}

Abbreviations: RFS, recurrence-free survival; $\mathrm{Cl}$, confidence interval; AFP, alpha-fetoprotein; NA, not applicable.

Cox regression analysis with the lowest AIC score identified predictors for model-pre (ALBI grade, serum AFP, and tumor size) and model-post (ALBI grade, serum AFP, and tumor size, MVI, and liver cirrhosis) (Table 2). There were no significant violations of the proportional hazard assumption assessed by scaled Schoenfeld residuals across time. The two models were presented as nomograms (Figure 1) as well as offline calculator deposited on the Github website (https:// github.com/radgrady/Offline-Calculator.git).

The resulting model-pre and model-post achieved respective C-indexes of 0.698 [95\%confidence interval (CI), 0 .659-0.737] and $0.728(95 \% \mathrm{CI}, 0.691-0.765)$ in the training cohort, $0.673(95 \% \mathrm{CI}, 0.622-0.724)$ and 0.713 (95\% CI, 0.661-0.765) in the validation cohort. The discriminatory performance of the two models was superior to that of BCLC stage (respective C-indexes of 0.610 and 0.595), HKLC stage (0.623 and 0.630), CLIP classification (0.640 and 0.634), and AJCC TNM system (0.684 and 0.623)
(Table 3). Calibration plot for the training cohort demonstrated good agreement between model-predicted and Kaplan-Meier-observed RFS; this result was confirmed in the validation cohort (Figure 2A). The tdAUC analysis also confirmed that the two proposed models improved prediction of HCC recurrence compared with HCC staging systems at different time points (Figure 2B and Table 3). The prediction error curves graphically showed the better performance of the two proposed models compared with HCC staging systems at various time points (Figure 2C). The model-pre and model-post yielded respective IBSs of 0.188 and 0.172 in the training cohort, 0.186 and 0.169 in the validation cohort, indicating lower prediction errors than HCC staging systems (Table 3). In both cohorts, DCA showed that the two proposed models provided larger net benefit compared with HCC staging systems and simple strategies (ie, follow-up of all patients or no patients) across the majority of reasonable range of threshold probabilities (Figure 3).

B

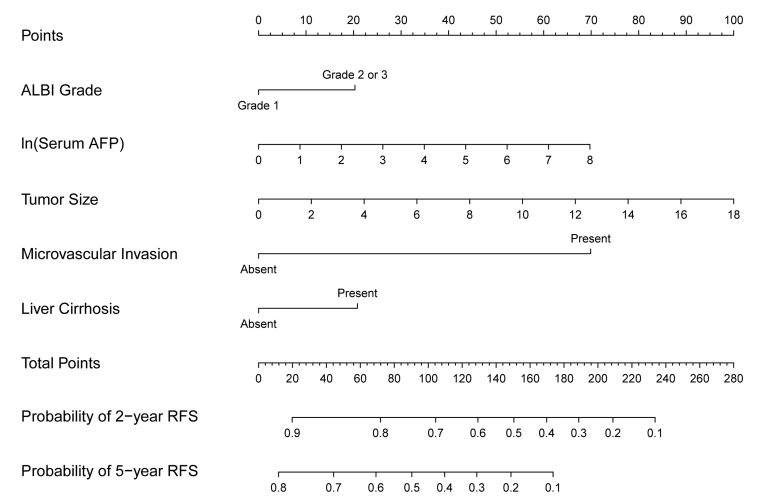

Figure I Pre-operative (A) and postoperative (B) models implemented as nomograms for predicting RFS. Abbreviations: ALBI, albumin-bilirubin; AFP, alpha-fetoprotein; RFS, recurrence-free survival. 
Table 3 Performance of the Two Models Compared with Staging Systems

\begin{tabular}{|l|l|l|l|l|l|l|}
\hline \multirow{2}{*}{ Model } & \multicolumn{2}{|l|}{ Training Cohort } & \multicolumn{2}{l|}{ Validation Cohort } \\
\cline { 2 - 7 } & C-index (95\% Cl) & tdAUC & IBS & C-index (95\% CI) & tdAUC & IBS \\
\hline Model-pre & $0.698(0.659-0.737)$ & 0.727 & 0.188 & $0.673(0.622-0.724)$ & 0.730 & 0.186 \\
Model-post & $0.728(0.691-0.765)$ & 0.783 & 0.172 & $0.713(0.661-0.765)$ & 0.779 & 0.169 \\
BCLC stage & $0.610(0.458-0.762)$ & 0.528 & 0.217 & $0.595(0.427-0.763)$ & 0.530 & 0.214 \\
HKLC stage & $0.623(0.548-0.698)$ & 0.588 & 0.211 & $0.630(0.529-0.730)$ & 0.593 & 0.211 \\
CLIP classification & $0.640(0.575-0.706)$ & 0.601 & 0.211 & $0.634(0.508-0.738)$ & 0.613 & 0.207 \\
AJCC TNM (8th) & $0.684(0.598-0.770)$ & 0.587 & 0.210 & $0.623(0.508-0.738)$ & 0.553 & 0.216 \\
\hline
\end{tabular}

Note: The tdAUC represented the median value of AUCs at various time points.

Abbreviations: $\mathrm{C}$-index, concordance index; $\mathrm{Cl}$, confidence interval; tdAUC, time-dependent area under the receiver operating characteristic curve; IBS, integrated Brier score; BCLC, Barcelona Clinic Liver Cancer; HKLC, Hong Kong Liver Cancer; CLIP, Cancer of the Liver Italian Program; AJCC, American Joint Committee on Cancer; TNM, tumor-node-metastasis.

\section{Recurrence Risk Stratification}

Using X-tile plot of the training cohort to generate the optimum cutoff score for model-pre, we assigned patients with model-pre score $>1.51$ (total point $>61$ in nomogram) to highrisk group and other patients to low-risk group (Supplementary Figure S2). In the training cohort, high-risk group was significantly associated with early HCC recurrence (median RFS, 22.9 months), compared with low-risk group (median RFS, 95.1 months; Log rank test, $P<0.001$ ). The model-pre could then subdivide patients from the independent validation cohort into high-risk group (median RFS, 30.0 months) and low-risk group (median RFS, 98.7 months; Log rank test, $P<0.001$ ) (Figure 4 and Table 4 ). Furthermore, recurrence pattern and corresponding treatment were significantly different between two risk categories (all $P<0.05$ ). In brief, extrahepatic recurrence was more frequently detected in high-risk group compared with low-risk group; a larger proportion of high-risk patients received systemic therapy or no treatment for recurrent HCC compared with low-risk patients (Table 5).

Similar results were achieved for model-post using 2.44 (total of 169 points in nomogram) as cutoff score. The model-post was able to identify $15 \%$ of patients at high-risk $(80.0 \%)$ of early recurrence (within 2 years after surgery) in both the training and validation cohorts (Table 4). Both study cohorts could be categorized into two separate risk groups with distinct recurrence patterns and corresponding treatments by the model-post (Figure 4, Tables 4 and 5).

\section{Discussion}

In this multi-institutional study, we built and validated two statistical models (model-pre and model-post) to predict disease recurrence for surgically treated patients with solitary HCC. The two models that enabled risk assessment of HCC recurrence before and after resection exhibited superior discrimination, with C-index of 0.673-0.728, lower prediction error, with IBS of 0.169-0.188, and larger net benefits, as determined by DCA, compared with widely used staging systems. Both models were capable of stratifying patients into two recurrence risk categories with distinct recurrence patterns, therefore facilitating treatment decision-making for patients with solitary HCC.

The definition of distinct prognostic subgroups of HCC patients may be helpful in driving personalized therapeutic strategies. Unfortunately, the challenge remains in risk stratification for HCC recurrence after resection. Indeed, the majority of HCC staging systems, except for AJCC TNM system, are not derived from surgically treated patients and therefore exhibited limited predictive value for HCC recurrence, with $\mathrm{C}$-indices of less than 0.640 in this study, that are consistent with previous reports. ${ }^{5,6}$ Prediction of postoperative recurrence of $\mathrm{HCC}$ should incorporate metastasis from the original tumor as well as de novo tumor arising in the cirrhotic liver, which generally manifests as early and late recurrence by using 2 years as the cutoff time., According to HCC staging systems, tumor size and ChildPugh class, two crucial factors for assessing tumor burden and liver function before surgery, are prognostic determinants for solitary HCC without vascular invasion. However, a plethora of studies have demonstrated that tumor size is not a clear-cut limiting factor, but the risk of vascular invasion and dissemination increases with diameter. ${ }^{1,13}$ Whilst having been widely adopted as a convenient indicator, Child-Pugh class has little prediction power for the extent of liver dysfunction. ${ }^{1-3}$ Additionally, most surgically treated patients with HCC belong to Child-Pugh class A, which accounted for about $95 \%$ of patients in our current 


\section{Training Cohort}

A

B
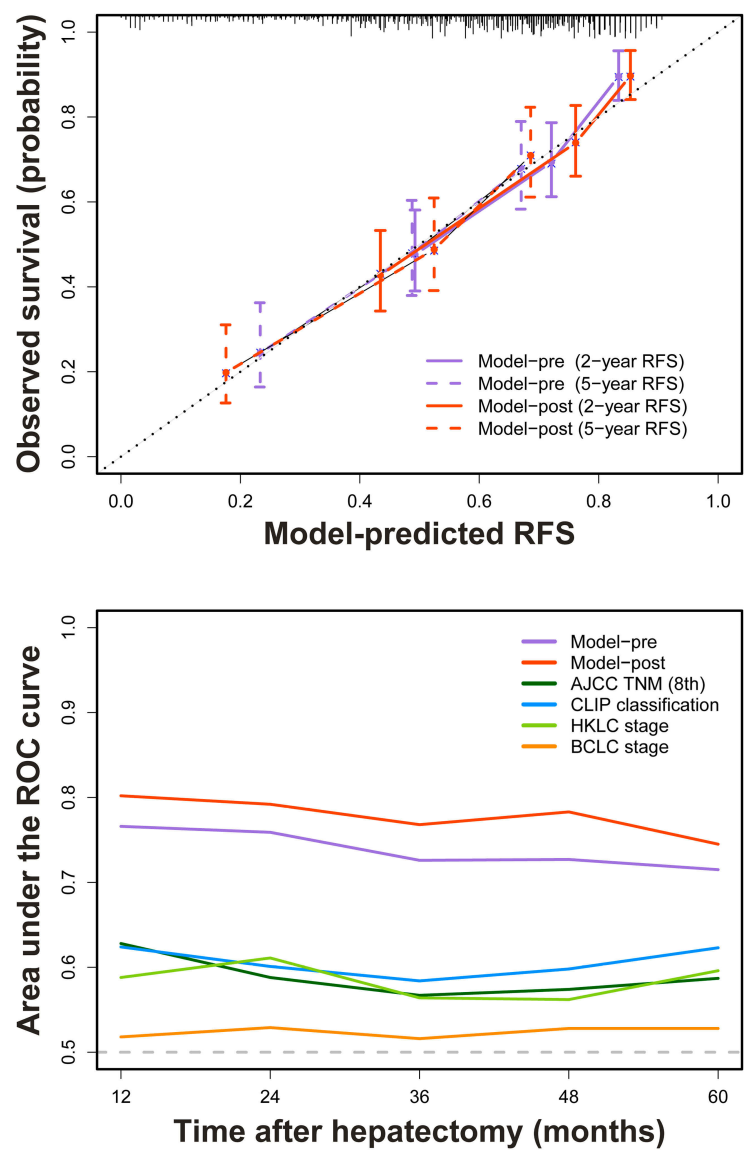

C

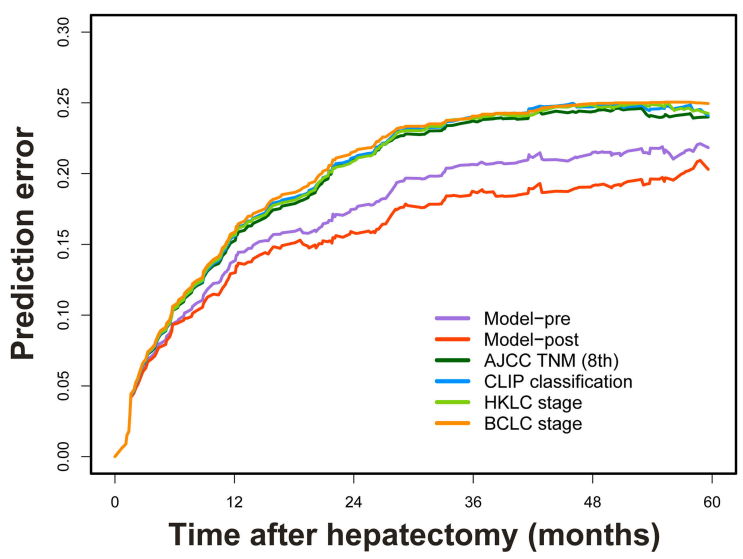

Validation Cohort
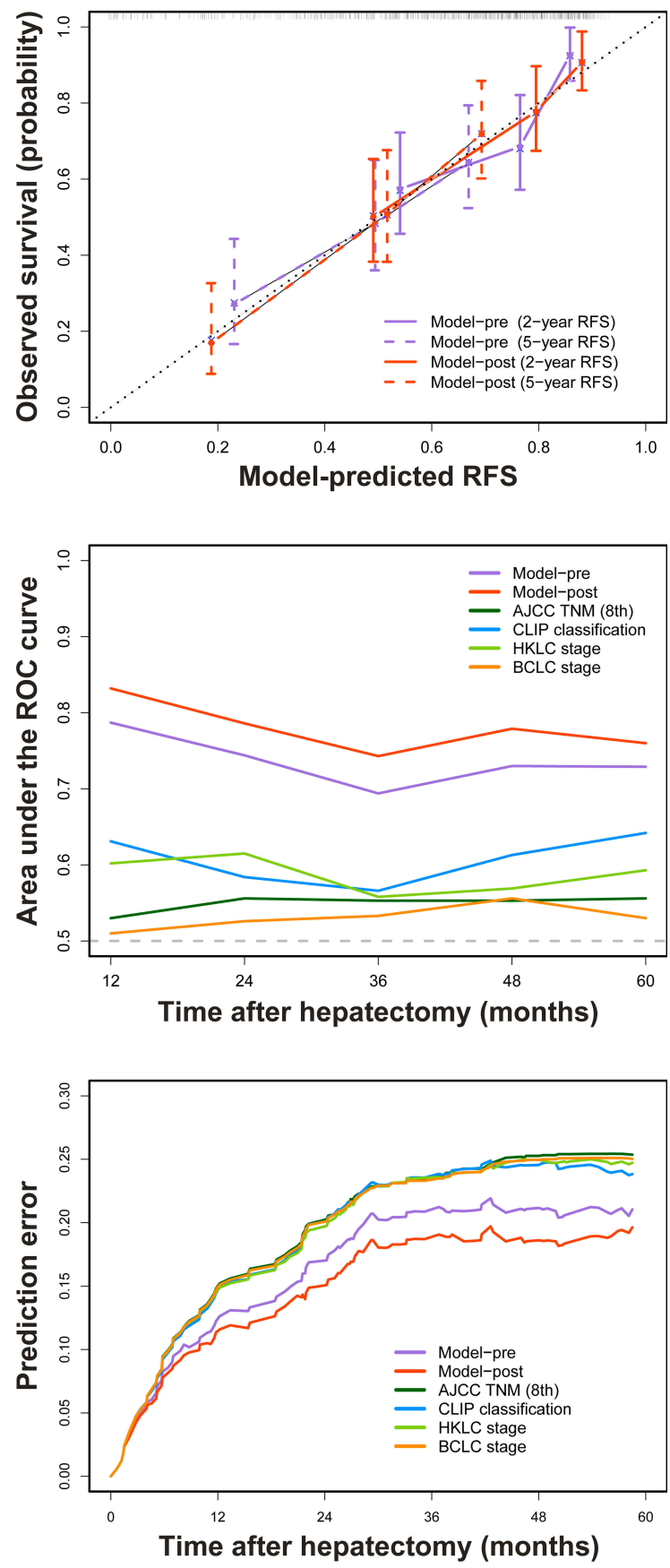

Figure 2 Calibration and performance of established models in the training and validation cohorts. (A) Calibration plots of two models. (B) Comparison of tdAUCs for established models and staging systems at various time points. (C) Comparison of prediction error estimates for established models and staging systems.

Abbreviations: RFS, recurrence-free survival; ROC, receiver operating characteristic; tdAUC, time-dependent area under the receiver operating characteristic curve; BCLC, Barcelona Clinic Liver Cancer; HKLC, Hong Kong Liver Cancer; CLIP, Cancer of the Liver Italian Program; AJCC, American Joint Committee on Cancer; TNM, tumor-node-metastasis.

datasets as well as previous publications. ${ }^{5,6}$ In comparison, ALBI grade is an objective and validated indicator of liver function that has been introduced as a prognostic factor for HCC; it is capable of satisfying patients classified as Child-
Pugh class A into distinct prognostic groups. ${ }^{5,8}$ For this reason, our results additionally indicate that ALBI grade is potentially more applicable in predicting disease recurrence after resection of solitary HCC. On the other hand, the 


\section{Training Cohort}
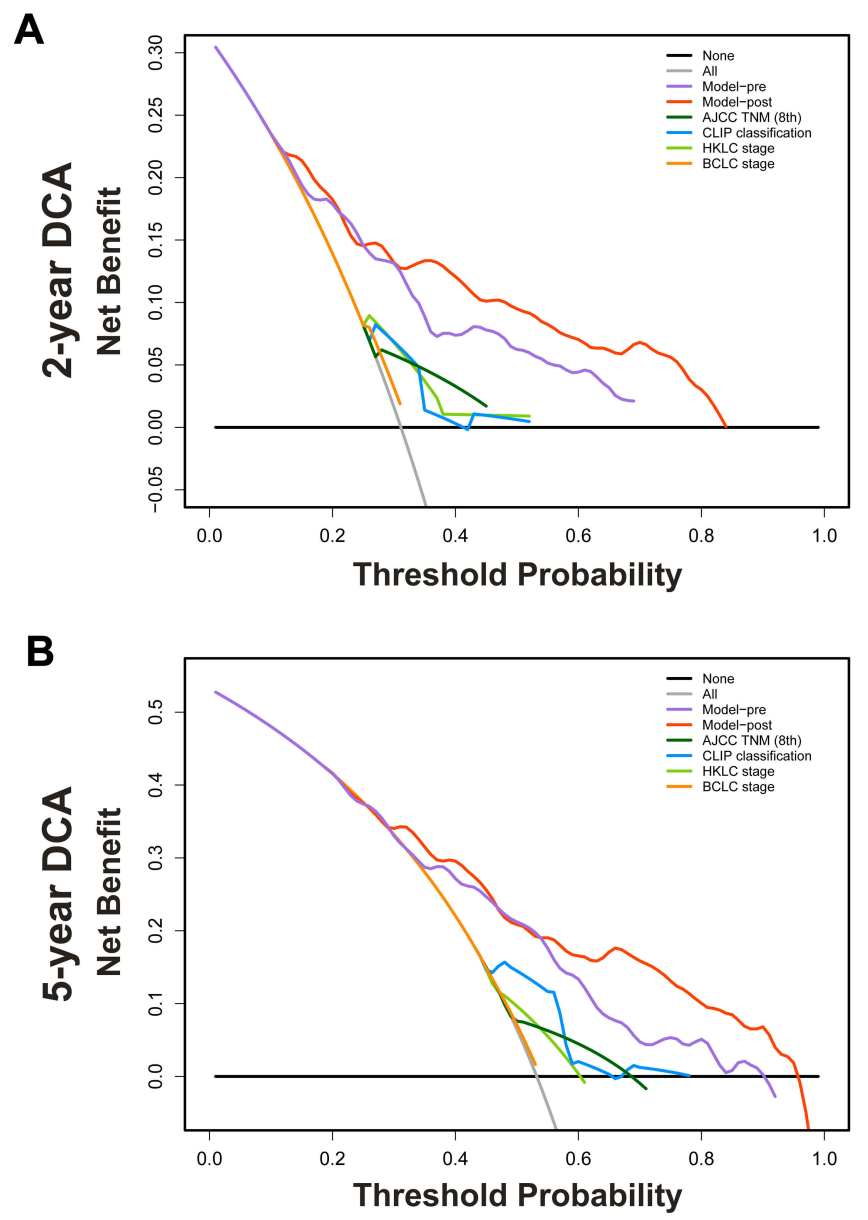

Validation Cohort
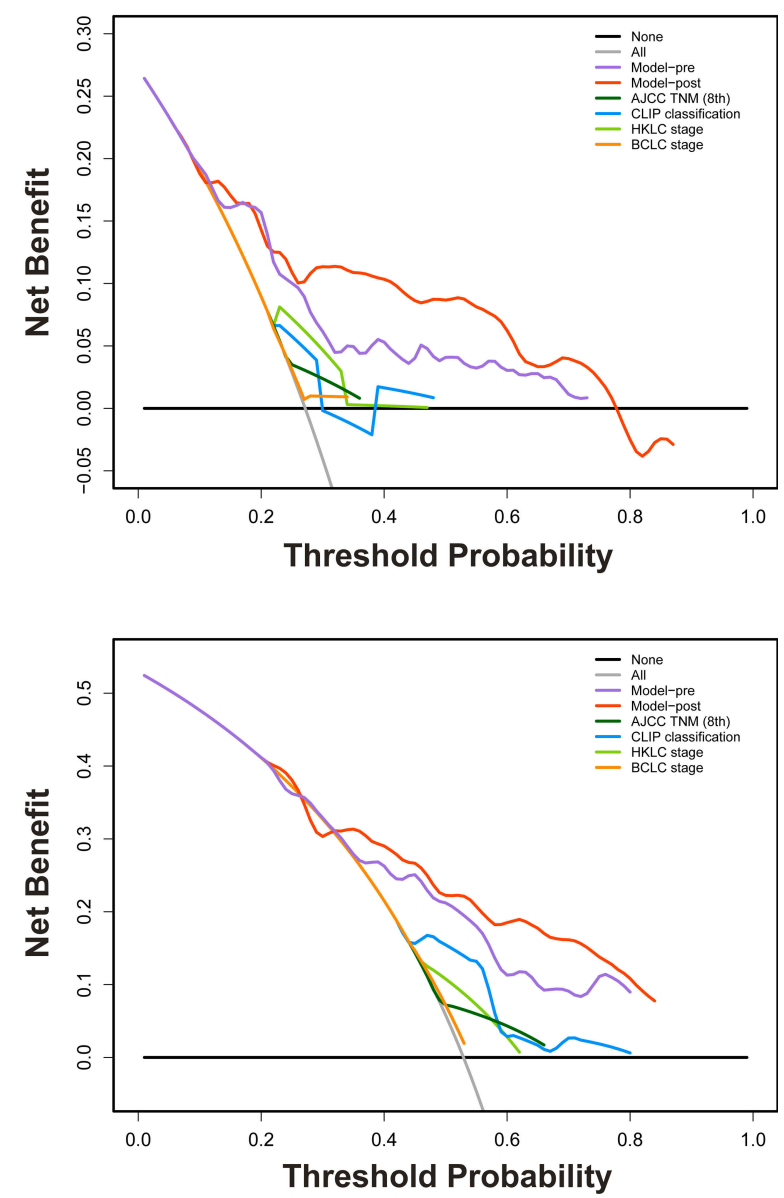

Figure 3 Decision curves for recurrence-free survival at $2(\mathbf{A})$ and $5(\mathbf{B})$ years using established models and staging systems in the training and validation cohorts. The $y$-axis measures the net benefit while the net benefit was calculated by summing the benefits (true-positive results) and subtracting the harms (false-positive results), weighting the latter by a factor related to the relative harm of an undetected tumor compared with the harm of unnecessary treatment. Both proposed models provided the highest net benefit compared with staging system, and simple strategies, such as follow-up of all patients or no patients, across the majority of the range of reasonable threshold probabilities at which a patient would choose to undergo imaging follow-up.

Abbreviations: DCA, decision curve analysis; BCLC, Barcelona Clinic Liver Cancer; HKLC, Hong Kong Liver Cancer; CLIP, Cancer of the Liver Italian Program; AJCC, American Joint Committee on Cancer; TNM, tumor-node-metastasis.

presence of MVI in resected specimen is a well-established risk factor for recurrent $\mathrm{HCC}$ after resection and an important component of pathology-based 8th edition AJCC staging to stratify patients with solitary HCC into distinct prognostic subgroups. ${ }^{10}$ As expected, it is a key variable in our postoperative model.

For simplicity and better acceptance, we developed the models by using easily ascertainable clinical parameters and multi-institutional databases. Of note, our two models share similar determinants with the Early Recurrence After Surgery for Liver tumor models, which were proposed for surgical patients across all HCC stages. ${ }^{5}$ This finding implies that our proposed models are robust for recurrence prediction in clinical practice. Promisingly, further use of our models might alter the surgical strategy for solitary HCC by allowing reappraisal of tumor biology before resection. For example, by using the pre-operative model, those candidates at high risk of early recurrence before surgery may need wider resection margins or anatomical liver resection to suppress potential tumor spread. ${ }^{3,14}$ In recent years, the policy of resection first followed by liver transplantation before recurrence detection permits an optimal use of the limited pool of organs and provides an excellent long-term outcome for patients at high risk of recurrence. ${ }^{1,15}$ Our postoperative model allows preemptive enlistment of high-risk patients for salvage liver transplant before the recurrence is identified. In addition, our models have important implications for individualized follow-up protocols. Specifically, 


\section{Training Cohort}

A A

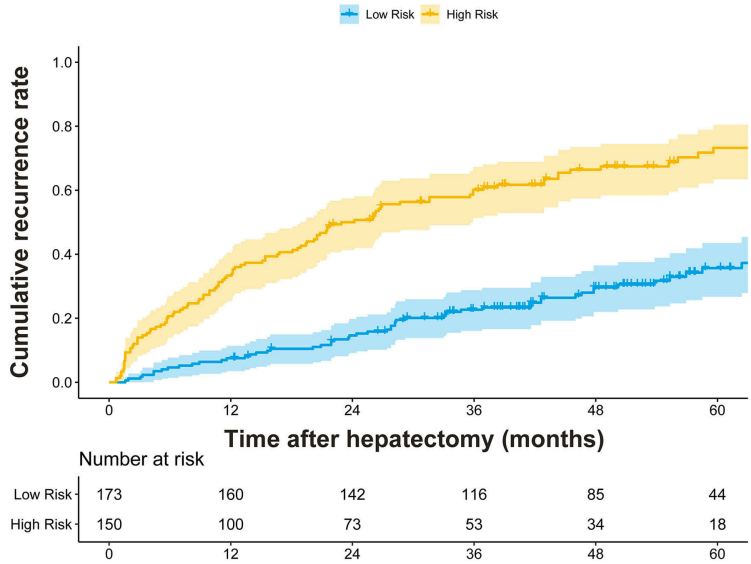

B
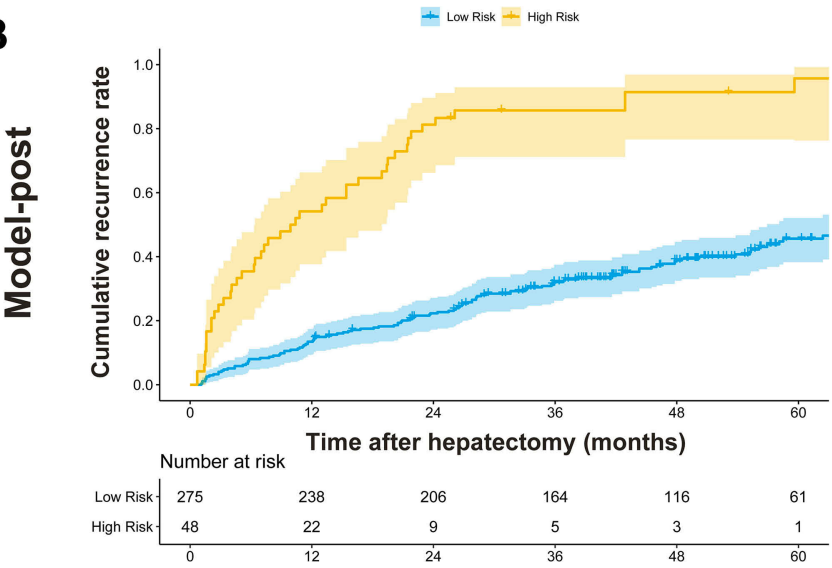

Validation Cohort
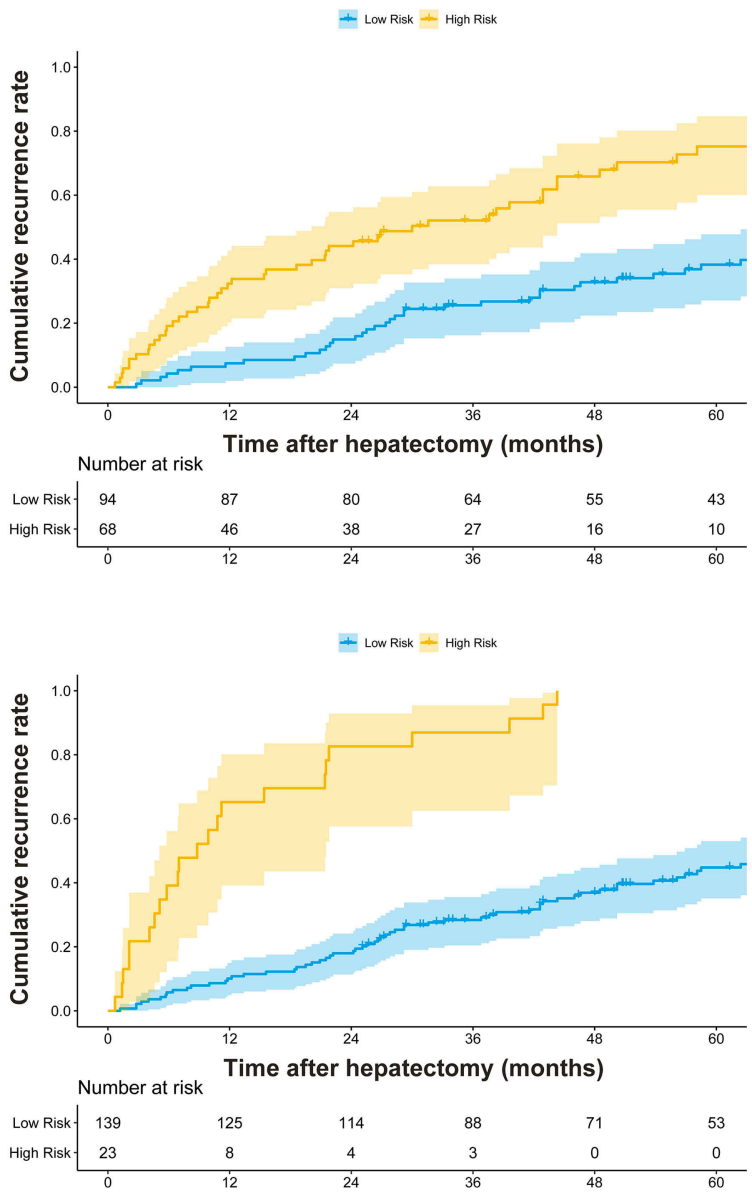

Figure 4 Cumulative rates of tumor recurrence stratified by the risk classification according to the model-pre (A) and model-post (B) in the training and validation cohorts. Shaded areas represent $95 \%$ confidence intervals.

low-risk patients may need less intensive follow-up, even within the first 2 years after resection, given their 2-year cumulative recurrence rate of $14-22 \%$, whereas high-risk patients may need intensive surveillance lasting for 5 years; high-risk patients should also receive intensive screening for distant metastasis since up to $23-39 \%$ of recurrent tumors involved extrahepatic sites. Accordingly, our two models may potentially offer clinical value in personalized treatment and surveillance for patients with solitary HCC. Notably, we presented two corresponding nomograms that provide graphical computation of recurrence risk for clinical use, accompanied by the offline calculator (https://github.com/ radgrady/Offline-Calculator.git) that enables surgical patients with solitary HCC to be easily managed and monitored on an individual basis.

Our study has several limitations. The main limitation of this study is its retrospective nature that could potentially introduce selection biases; future prospective studies are warranted to validate our models. A second limitation concerns that our models are not perfectly accurate; they merely provide improvement over the widely adopted staging systems in predicting HCC recurrence. Another limitation is that we did not investigate the molecular or genomic differences between distinct prognostic groups; this analysis might further facilitate individualized management of HCC, especially for high-risk patients.

\section{Conclusions}

In conclusion, we have developed and validated two models that allow individualized assessment of recurrence risk before and after resection in patients with solitary $\mathrm{HCC}$ at an early stage. The two models are powerful tools and appear to provide improvement over existing staging systems for recurrence prediction. The two models should be useful for pre-surgical counseling, clinical trial design, and 
Table 4 Median RFS and Cumulative Recurrence Rate According to Each Risk Group Defined by Two Models

\begin{tabular}{|c|c|c|c|c|c|c|c|c|}
\hline Model & Cohort & Group & $\mathbf{N}$ & $\begin{array}{l}\text { Median RFS, } \\
\text { Months }(95 \% \\
\text { Cl) }\end{array}$ & $\begin{array}{l}\text { 2-Year Recurrence } \\
\text { Rate, \% }(95 \% \mathrm{Cl})\end{array}$ & $\begin{array}{l}\text { 5-Year Recurrence } \\
\text { Rate, \% }(95 \% \mathrm{Cl})\end{array}$ & $\begin{array}{l}\text { Hazard Ratio } \\
(95 \% \mathrm{Cl})\end{array}$ & P value* \\
\hline \multirow{4}{*}{$\begin{array}{l}\text { Model- } \\
\text { pre }\end{array}$} & Training & Low-risk & 173 & 95.1 (82.4-NA) & $14.6(9.2-19.8)$ & $35.7(26.7-43.5)$ & Reference & \\
\hline & & High-risk & 150 & $22.9(18.9-35.9)$ & & & $3.346(2.421-4.624)$ & $<0.001$ \\
\hline & Validation & Low-risk & 94 & 98.7 (63.3-NA) & $\mid 4.9(7.4-2 \mid .8)$ & $38.3(27.1-47.8)$ & Reference & \\
\hline & & High-risk & 68 & $30.0(20.1-44.3)$ & $44.1(31.0-54.8)$ & $75.2(60.1-84.6)$ & $3.202(2.020-5.075)$ & $<0.001$ \\
\hline \multirow{4}{*}{$\begin{array}{l}\text { Model- } \\
\text { post }\end{array}$} & Training & Low-risk & 275 & 73.9 (56.9-NA) & $22.3(17.2-27.1)$ & $45.6(38.3-52.1)$ & Reference & \\
\hline & & High-risk & 48 & $10.2(6.4-18.9)$ & $81.3(66.2-89.6)$ & $95.7(76.3-99.2)$ & $24.99(13.52-46.20)$ & $<0.001$ \\
\hline & Validation & Low-risk & 139 & 82.4 (56.9-NA) & I8.0 (II.3-24.I) & $44.8(35.1-53.0)$ & Reference & \\
\hline & & High-risk & 23 & $8.8(5 .|-2| .5)$ & $82.6(57.6-92.9)$ & 100.0 (NA-NA) & 83.81 (32.07-219.0) & $<0.001$ \\
\hline
\end{tabular}

Note: *Calculated by using the Log rank test.

Abbreviations: RFS, recurrence-free survival; $\mathrm{Cl}$, confidence interval; NA, not applicable.

Table 5 HCC Recurrence Pattern and Treatment

\begin{tabular}{|c|c|c|c|c|c|c|c|c|c|c|c|c|}
\hline \multirow[t]{3}{*}{ Variable } & \multicolumn{6}{|c|}{ Training Cohort $(n=161)$} & \multicolumn{6}{|c|}{ Validation Cohort $(n=87)$} \\
\hline & \multicolumn{3}{|c|}{ Model-Pre } & \multicolumn{3}{|c|}{ Model-Post } & \multicolumn{3}{|c|}{ Model-Pre } & \multicolumn{3}{|c|}{ Model-Post } \\
\hline & $\begin{array}{l}\text { Low- } \\
\text { Risk } \\
(n=58)\end{array}$ & $\begin{array}{l}\text { High- } \\
\text { Risk } \\
(103)\end{array}$ & $P$ value & $\begin{array}{l}\text { Low-Risk } \\
(n=\mid 17)\end{array}$ & $\begin{array}{l}\text { High- } \\
\text { Risk } \\
(n=44)\end{array}$ & $P$ value & $\begin{array}{l}\text { Low- } \\
\text { Risk } \\
(n=39)\end{array}$ & $\begin{array}{l}\text { High- } \\
\text { Risk } \\
(n=48)\end{array}$ & $P$ value & $\begin{array}{l}\text { Low- } \\
\text { Risk } \\
(n=64)\end{array}$ & $\begin{array}{l}\text { High- } \\
\text { Risk } \\
(n=23)\end{array}$ & $P$ value \\
\hline $\begin{array}{l}\text { Site of } \\
\text { recurrence }\end{array}$ & & & 0.012 & & & 0.028 & & & 0.038 & & & 0.002 \\
\hline Intrahepatic only & $55(94.8)$ & $79(76.6)$ & & $103(88.0)$ & $31(70.4)$ & & $37(94.8)$ & $36(75.0)$ & & $59(92.2)$ & $14(60.9)$ & \\
\hline Extrahepatic only & $2(3.5)$ & $12(11.7)$ & & $7(6.0)$ & $7(15.9)$ & & I (2.6) & $9(18.8)$ & & $4(6.3)$ & $6(26.1)$ & \\
\hline $\begin{array}{l}\text { Intra- and } \\
\text { extrahepatic }\end{array}$ & $\mathrm{I}(1.7)$ & $12(11.7)$ & & $7(6.0)$ & $6(13.6)$ & & I (2.6) & $3(6.2)$ & & $I(1.5)$ & $3(13.0)$ & \\
\hline $\begin{array}{l}\text { Treatment of } \\
\text { recurrence }\end{array}$ & & & 0.006 & & & 0.003 & & & 0.013 & & & 0.023 \\
\hline $\begin{array}{l}\text { Surgical } \\
\text { resection/SLT }\end{array}$ & $19(32.8)$ & $16(15.5)$ & & $25(21.3)$ & $10(22.8)$ & & $13(33.3)$ & $6(12.5)$ & & $15(23.4)$ & $4(17.4)$ & \\
\hline Systemic therapy & $0(0.0)$ & $7(6.8)$ & & I (0.9) & $6(13.6)$ & & $0(0.0)$ & $7(14.6)$ & & $2(3.1)$ & $5(21.7)$ & \\
\hline $\begin{array}{l}\text { Other (ablation/ } \\
\text { radiation/TACE) }\end{array}$ & $36(62.0)$ & $64(62.2)$ & & $78(66.7)$ & $22(50.0)$ & & $24(61.6)$ & $30(62.5)$ & & $43(67.2)$ & II (47.8) & \\
\hline $\begin{array}{l}\text { No treatment/ } \\
\text { Supportive care }\end{array}$ & $3(5.2)$ & $16(15.5)$ & & $13(11.1)$ & $6(13.6)$ & & $2(5.1)$ & $5(10.4)$ & & $4(6.3)$ & $3(13.1)$ & \\
\hline
\end{tabular}

Note: Data are $n(\%)$.

Abbreviations: HCC, hepatocellular carcinoma; SLT, salvage liver transplantation; TACE, transarterial chemoembolization.

follow-up scheduling that may facilitate individualized management of patients with this disease.

\section{Abbreviations}

HCC, hepatocellular carcinoma; BCLC, Barcelona Clinic Liver Cancer; HKLC, Hong Kong Liver Cancer; CLIP, Cancer of the Liver Italian Program; AJCC, American Joint Committee on Cancer; TNM, tumor-nodemetastasis; TCIA, The Cancer Imaging Archive; ALBI, albumin-bilirubin; MVI, microvascular invasion; AFP, alpha-fetoprotein; RFS, recurrence-free survival; AIC, Akaike information criteria; C-index, concordance index; tdAUC, time-dependent area under the receiver operating characteristic curve; IBS, integrated Brier score; DCA, decision curve analysis; CI, confidence interval.

\section{Data Sharing Statement}

The data that support the findings of this study are available from the corresponding author upon reasonable request. 


\section{Author Contributions}

All authors contributed to data analysis, drafting or revising the article, gave final approval of the version to be published, and agree to be accountable for all aspects of the work.

\section{Funding}

This study was supported by the Key Program of the National Natural Science Foundation of China (31930020), the National Natural Science Foundation of China (81530048, 81470901, 81670570), and the Key Research and Development Program of Jiangsu Province (BE2016789).

\section{Disclosure}

The authors declare no potential conflicts of interest in this work.

\section{References}

1. Forner A, Reig M, Bruix J. Hepatocellular carcinoma. Lancet. 2018;391(10127):1301-1314. doi:10.1016/S0140-6736(18)30010-2

2. Villanueva A, Longo DL. Hepatocellular carcinoma. $N$ Engl J Med. 2019;380(15):1450-1462. doi:10.1056/NEJMra1713263

3. Galle PR, Forner A, Llovet JM, European Association for the Study of the Liver. EASL clinical practice guidelines: management of hepatocellular carcinoma. $J$ Hepatol. 2018;69(1):182-236. doi:10.10 16/j.jhep.2018.03.019

4. Qiu J, Peng B, Tang Y, et al. CpG methylation signature predicts recurrence in early-stage hepatocellular carcinoma: results from a multicenter study. J Clin Oncol. 2017;35(7):734-742. doi:10.12 00/JCO.2016.68.2153

5. Chan AWH, Zhong J, Berhane S, et al. Development of pre and post-operative models to predict early recurrence of hepatocellular carcinoma after surgical resection. J Hepatol. 2018;69(6):1284-1293. doi:10.1016/j.jhep.2018.08.027
6. Shim JH, Jun MJ, Han S, et al. Prognostic nomograms for prediction of recurrence and survival after curative liver resection for hepatocellular carcinoma. Ann Surg. 2015;261(5):939-946. doi:10.1097/ SLA.0000000000000747

7. Shen J, He L, Li C, et al. Nomograms to predict the individual survival of patients with solitary hepatocellular carcinoma after hepatectomy. Gut Liver. 2017;11(5):684-692. doi:10.5009/gnl16 465

8. Johnson PJ, Berhane S, Kagebayashi C, et al. Assessment of liver function in patients with hepatocellular carcinoma: a new evidence-based approach-the ALBI grade. J Clin Oncol. 2015;33 (6):550-558. doi:10.1200/JCO.2014.57.9151

9. Roayaie S, Blume IN, Thung SN, et al. A system of classifying microvascular invasion to predict outcome after resection in patients with hepatocellular carcinoma. Gastroenterology. 2009;137(3):85 0-855. doi:10.1053/j.gastro.2009.06.003

10. Vogel A, Cervantes A, Chau I, et al. Hepatocellular carcinoma: ESMO clinical practice guidelines for diagnosis, treatment and follow-up. Ann Oncol. 2018;29(Supp14):iv238-iv255. doi:10.1093/ annonc/mdy 308

11. Cohen ME, Ko CY, Bilimoria KY, et al. Optimizing ACS NSQIP modeling for evaluation of surgical quality and risk: patient risk adjustment, procedure mix adjustment, shrinkage adjustment, and surgical focus. J Am Coll Surg. 2013;217(2):336-46.e1. doi:10.10 16/j.jamcollsurg.2013.02.027

12. Camp RL, Dolled-Filhart M, Rimm DL. X-tile: a new bio-informatics tool for biomarker assessment and outcome-based cut-point optimization. Clin Cancer Res. 2004;10(21):7252-7259. doi:10.11 58/1078-0432.CCR-04-0713

13. Fuks D, Dokmak S, Paradis V, et al. Benefit of initial resection of hepatocellular carcinoma followed by transplantation in case of recurrence: an intention-to-treat analysis. Hepatology. 2012;55 (1):132-140. doi:10.1002/hep.24680

14. Agrawal S, Belghiti J. Oncologic resection for malignant tumors of the liver. Ann Surg. 2011;253(4):656-665. doi:10.1097/SLA.0b013e3 $181 \mathrm{fc} 08 \mathrm{ca}$

15. Ferrer-Fàbrega J, Forner A, Liccioni A, et al. Prospective validation of ab initio liver transplantation in hepatocellular carcinoma upon detection of risk factors for recurrence after resection. Hepatology. 2016;63(3):839-849. doi:10.1002/hep.28339

\section{Publish your work in this journal}

Cancer Management and Research is an international, peer-reviewed open access journal focusing on cancer research and the optimal use of preventative and integrated treatment interventions to achieve improved outcomes, enhanced survival and quality of life for the cancer patient.
The manuscript management system is completely online and includes a very quick and fair peer-review system, which is all easy to use. Visit http://www.dovepress.com/testimonials.php to read real quotes from published authors. 